NOTA

\title{
DESENVOLVIMENTO RADICULAR DO ALGODOEIRO EM RESPOSTA À LOCALIZAÇÃO DO FERTILIZANTE ${ }^{(1)}$
}

\author{
Fábio Suano de Souza ${ }^{(2)}$, Rogerio Farinelli ${ }^{(3)} \&$ Ciro Antonio Rosolem ${ }^{(4)}$
}

\begin{abstract}
RESUMO
A utilização de adubos pode-se tornar prejudicial caso o fertilizante não seja localizado adequadamente. No presente trabalho foram estudados o crescimento radicular do algodoeiro (Gossypium hirsutum), o crescimento inicial e a nutrição da planta, considerando o local de aplicação do fertilizante. $O$ estudo foi realizado em vasos com parede de vidro. O fertilizante foi colocado a $5,0 \mathrm{~cm}$ abaixo e $0,0,2,5$, 5,0 e $10,0 \mathrm{~cm}$ ao lado das sementes. $O$ crescimento radicular foi avaliado a cada três dias e, aos 21 dias após a emergência, as plantas foram coletadas, sendo avaliada a produção de matéria seca e a absorção de macronutrientes. A aplicação de fertilizante proporcionou crescimento inicial mais vigoroso do sistema radicular mesmo em solo previamente corrigido e adubado, o que é importante no estabelecimento da cultura. Somente houve bom crescimento inicial do sistema radicular e da parte aérea do algodoeiro quando o fertilizante foi aplicado de 5,0 a $10,0 \mathrm{~cm}$ ao lado e $5,0 \mathrm{~cm}$ abaixo das sementes.
\end{abstract}

Termos de indexação: raízes, adubação, semeadura, Gossypium hirsutum.

\section{SUMMARY: COTTONROOT DEVELOPMENT AS AFFECTED BY FERTILIZER PLACEMENT}

Unless fertilizer is properly placed in the soil it can be harmful. This experiment was conducted to study cotton (Gossypium hirsutum) root growth and initial plant development and nutrition as affected by fertilizer placement. Cotton plants were grown in pots with a glass wall. The fertilizer was applied $5.0 \mathrm{~cm}$ under the seed row and $0,2.5,5.0$ and $10.0 \mathrm{~cm}$ beside the seed row. Root growth was evaluated every 3 days, and 21 days after emergence the plants were harvested. Dry matter production and macronutrient absorption were evaluated. Even in previously limed and fertilized soil, localized fertilizer application

\footnotetext{
${ }^{(1)}$ Recebido para publicação em outubro de 2006 e aprovado em janeiro de 2007.

(2) Pesquisador Instituto Agronômico do Paraná. Caixa Postal 481, CEP 86047-902 Londrina (PR). E-mail: fssouza@iapar.br

${ }^{(3)}$ Doutorando em Agronomia, Área de Agricultura, Universidade Estadual Paulista - FCA/UNESP. Caixa Postal 237, CEP 18603970 Botucatu (SP). E-mail: rfarinelli@fca.unesp.br

(4) Professor do Departamento de Produção Vegetal, FCA/UNESP. Bolsista do CNPq. E-mail: rosolem@fca.unesp.br
} 
reinforced the initial growth of cotton roots, which is very important for a good crop establishment in the field. Normal root growth and adequate initial plant development was only observed when the fertilizer was placed $5.0 \mathrm{~cm}$ below and from 5.0 to $10.0 \mathrm{~cm}$ distance from the seed row.

Index terms: roots, fertilization, sowing, Gossypium hirsutum.

\section{INTRODUÇÃO}

A absorção de nutrientes pode ser limitada tanto pelo menor crescimento do sistema radicular como também pela redução da atividade radicular causada por fatores físicos e químicos do solo (Carvalho et al., 2001). O sistema radicular do algodoeiro cresce em comprimento até a época de pleno florescimento (Nayakekorala \& Taylor, 1990), ocorrendo, após esse período, somente incremento na matéria seca das raízes. Por isso, trata-se de um sistema radicular pouco volumoso, que explora pobremente certas regiões do solo (Brounder \& Cassman, 1990).

A necessidade de adubo na fase inicial de crescimento da planta com pequeno crescimento radicular, que se observa nos primeiros 10 dias, é mínima (Silva, 1999), de modo que a aplicação de adubos pode se tornar prejudicial caso o fertilizante não seja aplicado adequadamente. A importância da aplicação de quantidades adequadas de nutrientes nas plantas é bem conhecida. No entanto, a resposta aos fertilizantes pode ser limitada pela localização imprópria, particularmente do ponto de vista da injúria dos sais às plântulas, uma das causas da diminuição da população de plantas (Tisdale \& Nelson, 1966). Foi observado aumento na freqüência de efeitos positivos da adubação potássica quando essa injúria foi evitada (Silva, 1999).

Um dos métodos de localização do adubo é em linha. $\mathrm{O}$ fertilizante pode ser aplicado no mesmo tubo da máquina, com a semente, como em semeadora de sementes pequenas, ou pode ser localizado no sulco com a semente. Quando doses maiores de fertilizantes são usadas, a emergência de plântulas é atrasada e, em alguns casos, a produção é reduzida, como mostram Tisdale \& Nelson (1966).

Amostragens a 15,5 e $31 \mathrm{~cm}$ de distância da linha de plantas de milho revelaram que o fertilizante nitrogenado aplicado entre as linhas de semeadura proporcionou maior densidade de raiz, na amostragem a $31 \mathrm{~cm}$, do que os tratamentos em que o $\mathrm{N}$ foi aplicado a lanço e aquele em que este elemento estava localizado próximo à semente, mostrando melhor distribuição de raízes no primeiro tratamento (Schröder et al., 1996).

Foi notada injúria à raiz do algodoeiro, com menor crescimento de raízes laterais, mesmo quando foram utilizadas doses da ordem de $50 \mathrm{~kg} \mathrm{ha}^{-1}$ de uma formulação 18-46-00, provavelmente em razão do alto teor de N (USDA, 2004).
Da mesma forma, houve diminuição significativa do crescimento da raiz principal e da iniciação de raízes laterais quando foram aplicados, no sulco, aproximadamente $100 \mathrm{~kg} \mathrm{ha}^{-1}$ das fórmulas $10-34-00$ ou 11-52-00. A aplicação de $200 \mathrm{~kg} \mathrm{ha}^{-1}$ dessas fórmulas no sulco de semeadura inibiu completamente o crescimento radicular.

É interessante notar que as doses empregadas no trabalho do USDA (2004) são menores que as usualmente empregadas nas lavouras brasileiras e não têm K, demonstrando o risco de danos ao sistema radicular do algodoeiro.

O objetivo deste trabalho foi avaliar localizações e distâncias de uma determinada quantidade de fertilizante, em relação à semente, em uma cultivar de algodão, durante a fase de estabelecimento da cultura.

\section{MATERIAL E MÉTODOS}

O experimento foi realizado em casa de vegetação na FCA/UNESP em Botucatu, SP, em minirrizotrons construídos com tubos de PVC de $30 \mathrm{~cm}$ de diâmetro interno, cortados ao meio no sentido longitudinal. No plano do corte foi colocado vidro de $5 \mathrm{~mm}$ de espessura, de modo que as raízes pudessem ser visualizadas. A janela de vidro foi coberta com uma placa de madeira deslizante. Os vasos foram preenchidos com solo corrigido, cuja análise química (Raij \& Quaggio, 1983) revelou $\mathrm{pH}_{\mathrm{CaCl}}$ de 5,6; 17,8 $\mathrm{g} \mathrm{kg}^{-1}$ de $\mathrm{MO} ; 92,5 \mathrm{mg} \mathrm{dm}^{-3}$ de $\mathrm{P}_{\text {resina }} ; 2,7,31,3$ e $12,1 \mathrm{mmol}_{\mathrm{c}} \mathrm{dm}^{-3} \mathrm{de} \mathrm{K}^{+}, \mathrm{Ca}^{2+} \mathrm{e}$ $\mathrm{Mg}^{2+}$, respectivamente; CTC de 67, $8 \mathrm{mmol}_{\mathrm{c}} \mathrm{dm}^{-3}$; e 68 \% de saturação por bases.

Foram utilizadas sementes de algodão (Gossypium hirsutum, var. Latifolia) da cultivar Delta Opal, prégerminadas por $36 \mathrm{~h}$ em câmara de germinação a $30{ }^{\circ} \mathrm{C}$. A semeadura foi realizada colocando-se as sementes próximas à placa de vidro do minirrizotrom.

Foi utilizado o fertilizante formulado 02-20-20, na dose correspondente a $500 \mathrm{~kg} \mathrm{ha}^{-1}$. O adubo foi aplicado em sulcos abertos nos vasos, perpendicularmente à parede de vidro, e a quantidade foi calculada de acordo com o comprimento do sulco. O fertilizante foi depositado no solo em cinco diferentes posições em relação à semente: adubo a $5 \mathrm{~cm}$ abaixo da semente; a $5 \mathrm{~cm}$ abaixo e $5 \mathrm{~cm}$ ao lado da semente; a $5 \mathrm{~cm}$ abaixo e $10 \mathrm{~cm}$ ao lado; a mesma dose do adubo dividida em $5 \mathrm{~cm}$ abaixo da semente e $2,5 \mathrm{~cm}$ de cada lado; a 
mesma dose do adubo dividida em $5 \mathrm{~cm}$ abaixo e $5 \mathrm{~cm}$ de cada lado; e testemunha, sem adubação.

O crescimento radicular foi avaliado a cada três dias, desenhando-se as raízes visíveis através do plástico transparente colocado sobre o vidro do minirrizotrom, utilizando-se canetas de cores diferentes para cada avaliação. No final do experimento, os plásticos foram colocados sobre uma grade de 1,0 x 1,0 cm e o comprimento radicular, para cada período, foi determinado usando-se o método de Tenant (1975). A produção de matéria seca foi avaliada aos 21 dias após a semeadura, quando as plantas foram coletadas e secas até atingirem peso constante. A matéria seca da parte aérea foi moída e foram analisados os teores de N, P e K, de acordo com recomendações de Malavolta et al. (1997).

O delineamento utilizado foi em blocos casualizados, com seis tratamentos e quatro repetições. Os resultados foram submetidos a análise de variância. As médias foram comparadas pelo teste de Tukey a $5 \%$. Equações foram ajustadas ao crescimento radicular, escolhendo-se o modelo significativo de maior coeficiente de determinação. A taxa de crescimento radicular foi calculada, obtendo-se a primeira derivada das curvas ajustadas aos resultados de crescimento radicular.

\section{RESULTADOS E DISCUSSÃO}

Verificou-se grande variação do comprimento radicular entre a testemunha e os tratamentos adubados, mostrando que, mesmo em solo corrigido, a presença de adubo próximo ao sistema radicular, durante os primeiros dias após a emergência, é importante para o bom estabelecimento da cultura de algodão, resultando em sistema radicular aproximadamente cinco vezes mais longo que no tratamento sem adubo, nos primeiros 21 dias após a semeadura (Figura 1). Há tendência, recente, de se evitar a aplicação de adubos por ocasião da semeadura, procurando-se melhorar o rendimento das máquinas. Entretanto, os resultados deste trabalho ratificam a necessidade de aplicação de fertilizantes por ocasião da semeadura, para o algodoeiro.

Os tratamentos com fertilizante aplicado $10,0 \mathrm{~cm}$ ao lado e 5,0 cm de cada lado apresentaram maiores valores de comprimento radicular (Figura 1). É interessante notar que, até os 15 dias, no tratamento com fertilizante a $5,0 \mathrm{~cm}$ de cada lado, havia menor comprimento de raízes, que se recuperou deste dia em diante. Isso provavelmente ocorreu porque as raízes ultrapassaram a zona mais crítica em termos de salinidade, encontrando boas condições de crescimento em seguida. Assim, de modo geral, o fertilizante deve ser colocado mais distante da semente, melhor, pelo menos até $10,0 \mathrm{~cm}$. Nos tratamentos em que o fertilizante foi colocado mais

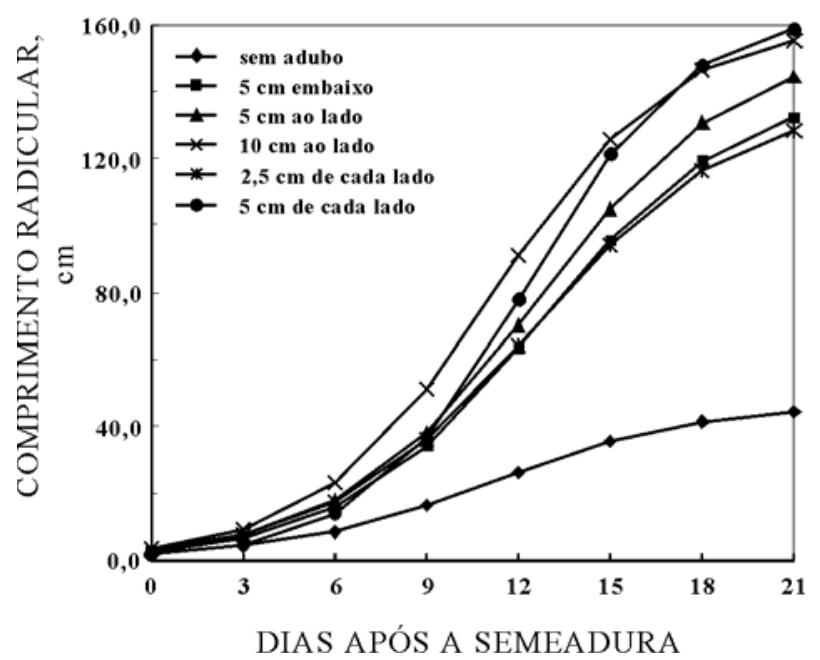

Figura 1. Comprimento radicular do algodoeiro de acordo com a localização do adubo em relação às sementes.

próximo à semente $(2,5 \mathrm{~cm}$ de cada lado e $5,0 \mathrm{~cm}$ diretamente abaixo da semente) verificaram-se menores valores de comprimento radicular. Provavelmente tal injúria ocorreu devido ao efeito salino do fertilizante, não sendo tão pronunciado em tratamentos em que este estava mais distante da semente (5,0 cm de cada lado da semente, por exemplo). De longa data se sabe que, se o sal estiver concentrado muito próximo à plântula, pode ocorrer prejuízo no crescimento (Tisdale \& Nelson, 1966). Outros autores, como Silva (1999), afirmam que a necessidade de fertilizante na fase inicial de crescimento radicular, que ocorre nos primeiros 10 dias, é mínima, podendose tornar prejudicial caso ele não seja aplicado adequadamente. Nos Estados Unidos (USDA, s.d.) foi notada injúria à raiz do algodoeiro mesmo com doses da ordem de $50 \mathrm{~kg} \mathrm{ha}^{-1}$ da formulação 18-46-00, provavelmente em razão do alto teor de N. No mesmo trabalho há relato de diminuição significativa do crescimento da raiz principal e da iniciação de raízes laterais quando foram aplicados, no sulco, aproximadamente $100 \mathrm{~kg} \mathrm{ha}^{-1}$ das fórmulas 10-34-00 ou 11-52-00. Assim, mesmo formulações que não sejam muito ricas em $\mathrm{N}$ e $\mathrm{K}$ podem causar redução significativa no crescimento radicular do algodoeiro, como foi o caso no presente trabalho.

Em geral, os efeitos dos tratamentos sobre a taxa de crescimento foram semelhantes aos observados no comprimento total de raízes (Figura 2). Entretanto, observou-se que a máxima taxa de crescimento radicular no tratamento sem adubo e com adubo a $10,0 \mathrm{~cm}$ ocorreu próximo aos 12 dias após a semeadura. Nos demais tratamentos, com fertilizante mais próximo à semente, a taxa máxima ocorreu um pouco mais tarde. Por outro lado, aos 21 dias esses efeitos foram exatamente inversos, ou seja, o tratamento sem adubo e o tratamento com fertilizante 
a 10,0 já apresentavam taxa de crescimento mais baixa que a dos demais. Assim, seria possível que, com o tempo e em condições favoráveis, pudesse ocorrer recuperação no comprimento total das raízes.

Foram ainda observadas diferenças importantes na quantidade de raízes e na sua distribuição no solo (Figura 3), em que fica muito claro não só o maior crescimento, como a melhor distribuição do sistema radicular do algodoeiro, quando o fertilizante foi colocado em duas faixas: a 5,0 cm de cada lado e 5,0 cm abaixo das sementes. É importante lembrar que, embora essa localização seja muito interessante do

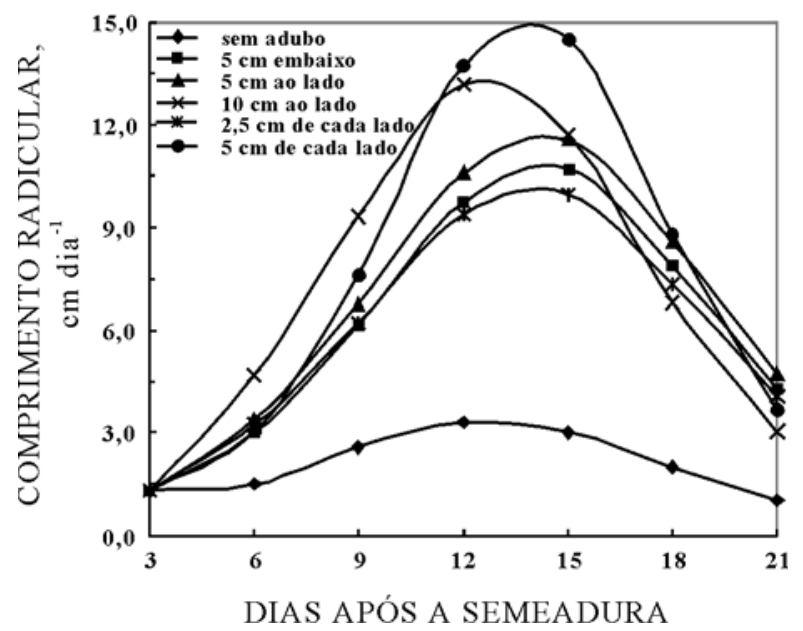

Figura 2. Taxa de crescimento das raízes de algodoeiro de acordo com o tempo e a localização do adubo. ponto de vista do crescimento radicular, é pouco prática em termos de uso no campo. Verifica-se que houve diferença significativa para a produção de matéria seca da parte aérea do algodoeiro (Quadro 1). Além da resposta ao adubo aplicado, entre os tratamentos com fertilizante, o adubo colocado $5,0 \mathrm{~cm}$ abaixo da linha de sementes resultou em menor produção que os demais tratamentos com fertilizante. A localização do fertilizante em relação à semente influenciou, além de comprimento radicular e produção de matéria seca, os teores de $\mathrm{N}$ e $\mathrm{P}$ da parte aérea das plantas. Entre os macronutrientes (Quadro 1), o $\mathrm{N}$ e o $\mathrm{P}$ apresentaram valores acima dos níveis críticos em todos os tratamentos (Malavolta, 1980). Para P, não houve diferença significativa entre os tratamentos. Quanto à absorção de nutrientes, apenas houve resposta nos teores de $\mathrm{N}$ e $\mathrm{P}$ do algodoeiro de acordo com a do fertilizante. Com relação à localização do adubo, não foram notados efeitos significativos.

Considerando-se o modo de contato entre os íons e as raízes das plantas, seria esperada correlação significativa entre os teores de $\mathrm{P}$ e K com o comprimento total das raízes, uma vez que esses nutrientes entram em contato com estas principalmente por difusão. Entretanto, isso não foi observado, provavelmente em razão de se estar trabalhando com um solo bem corrigido, tendo esse efeito sido mascarado.

Por sua vez, no final do período de observação, a taxa de crescimento radicular apresentou correlação significativa com o teor de $\mathrm{N}$ da parte aérea das plantas, não sendo o mesmo observado para as demais correlações (Quadro 2). O tratamento com fertilizante colocado 5,0 $\mathrm{cm}$ abaixo e 10,0 $\mathrm{cm}$ ao lado das sementes apresentou baixo teor de $\mathrm{N}$ e menor taxa de crescimento radicular nesse mesmo período.

Quadro 1. Produção de matéria seca e teores de nitrogênio, fósforo e potássio da parte aérea de plantas de algodão, de acordo com a localização do adubo

$\begin{array}{lllll}\text { Tratamento } & \text { Matéria seca } & \text { N } & \text { P } & \text { K }\end{array}$

\begin{tabular}{lcccc} 
& g/vaso & & \\
\cline { 3 - 4 } Testemunha & $0,09 \mathrm{c}^{(1)}$ & $35,2 \mathrm{c}$ & $1,3 \mathrm{~b}$ & 16,1 \\
$5 \mathrm{~cm}$ abaixo & $0,19 \mathrm{~b}$ & $47,1 \mathrm{ab}$ & $1,8 \mathrm{a}$ & 16,7 \\
$5 \mathrm{~cm}$ ao lado & $0,41 \mathrm{a}$ & $50,7 \mathrm{a}$ & $1,6 \mathrm{a}$ & 12,2 \\
$10 \mathrm{~cm}$ ao lado & $0,38 \mathrm{a}$ & $44,8 \mathrm{ab}$ & $1,6 \mathrm{a}$ & 13,4 \\
$2,5 \mathrm{~cm}$ de cada lado & $0,39 \mathrm{a}$ & $44,3 \mathrm{ab}$ & $1,5 \mathrm{ab}$ & 15,8 \\
$5 \mathrm{~cm}$ de cada lado & $0,49 \mathrm{a}$ & $45,0 \mathrm{ab}$ & $1,4 \mathrm{ab}$ & 11,7 \\
Média & 0,32 & 44,0 & 1,46 & 14,3 \\
Teste F & $2,48^{\mathrm{ns}}$ & $9,33^{* *}$ & $5,57^{* *}$ & $2,35^{\mathrm{ns}}$ \\
CV (\%) & 25,1 & 7,8 & 14,0 & 19,4
\end{tabular}

(1) Letras diferentes nas colunas indicam diferença significativa pelo teste de Tukey $(\mathrm{P}<0,05)$.

** Teste $\mathrm{F}$ significativo $(\mathrm{P}<0,001)$; ns: teste $\mathrm{F}$ não-significativo. 
Quadro 2. Correlações entre comprimento total de raízes e taxa de crescimento radicular e teores de nitrogênio, fósforo, potássio e matéria seca do algodoeiro, para os resultados obtidos a partir de 18 dias após a semeadura

\begin{tabular}{|c|c|c|c|c|}
\hline \multirow{2}{*}{ Parâmetro } & \multicolumn{4}{|c|}{ Correlação ( $\left.R^{2}\right)$} \\
\hline & $\mathbf{N}$ & $\mathbf{P}$ & $\mathbf{K}$ & Matéria seca \\
\hline Comprimento total de raízes no final do experimento & 0,55 & 0,07 & 0,38 & 0,42 \\
\hline Taxa de crescimento radicular no final do experimento & $0,91^{* *}$ & 0,11 & 0,03 & 0,22 \\
\hline
\end{tabular}

**: Significativo a $5 \%$.

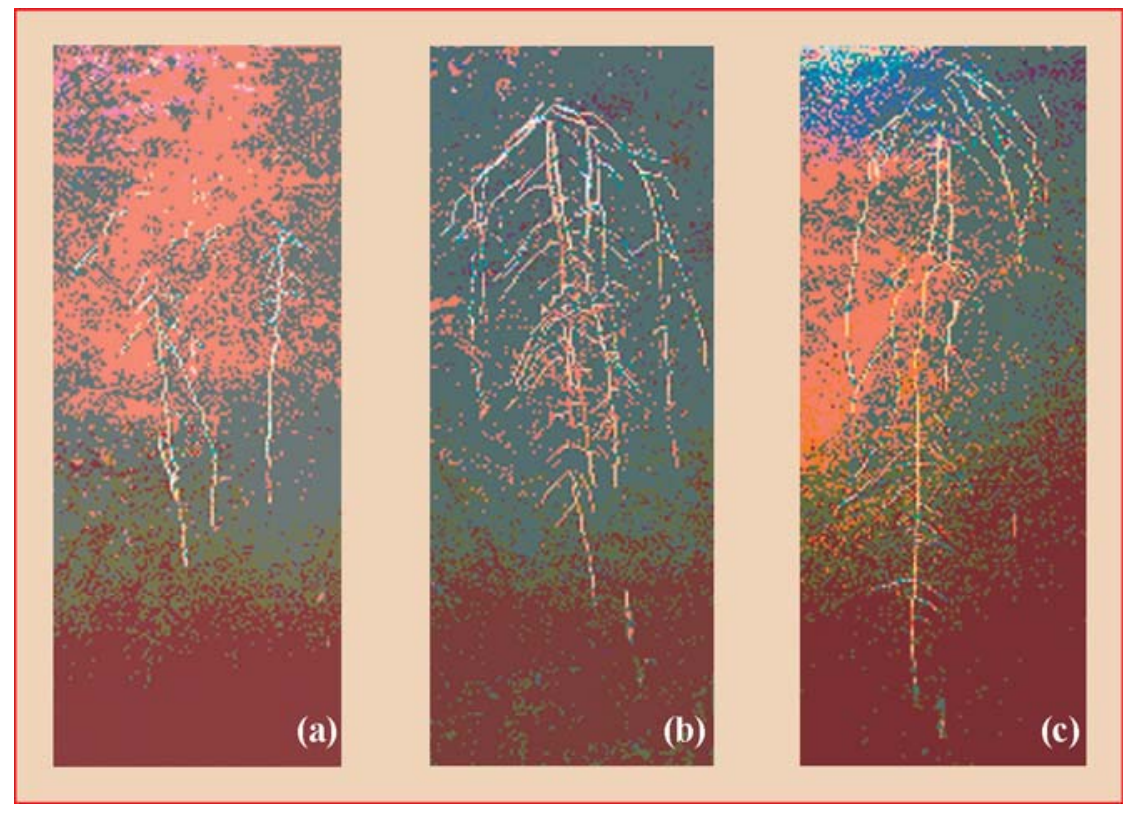

Figura 3. Sistema radicular com o tratamento com adubo $5 \mathrm{~cm}$ abaixo da semente (a), 5 cm de cada lado da semente (b) e $5 \mathrm{~cm}$ ao lado da semente (c).

\section{CONCLUSÕES}

1. A aplicação de fertilizante por ocasião da semeadura do algodoeiro é fundamental para que haja crescimento vigoroso e rápido do sistema radicular.

2. Para que haja bom crescimento inicial do sistema radicular e da parte aérea do algodoeiro, é importante que o fertilizante seja aplicado de 5,0 a $10,0 \mathrm{~cm}$ ao lado e $5,0 \mathrm{~cm}$ abaixo das sementes.

\section{LITERATURA CITADA}

BROUDER, S.M. \& CASSMAN, K.G. Root development of two cotton cultivars in relation to potassium uptake and plant growth in vermiculite soil. Field Crops Res., 23:187203, 1990.
CARVALHO, M.A.C.; PAULINO, H.B.; FURLANI JÚNIOR, E.; BUZETH, S.; SÁ, M.E. \& ATHAYDE, M.L.F. Uso da adubação foliar nitrogenada e potássica no algodoeiro. Bragantia, 60:239-244, 2001.

MALAVOLTA, E.; VITTI, G.C. \& OLIVEIRA, S.A. Avaliação do estado nutricional das plantas: Princípios e aplicações, 2.ed. Piracicaba, Potafós, 1997. 317p.

NAYAKEKORALA, H. \& TAYLOR, H.M. Phosphorus uptake rates of cotton roots at different growth stages from different soil layers. Plant Soil, 122:105-110, 1990.

RAIJ, B. van \& QUAGGIO, J.A. Métodos de análise de solos para fins de fertilidade. Campinas, Instituto Agronômico, 1983. 31p.

SCHRÖDER, J.J.; GROENWOLD, J. \& ZAHARIEVA, T. Soil mineral nitrogen availability to young maize plants as related to root lenght density distribution and fertilizer application method. Nether. J. Agric. Sci., 44:209-225, 1996. 
SILVA, N.M. Nutrição mineral e adubação do algodoeiro no Brasil. In: CIA, E.; FREIRE, E.C. \& SANTOS, W.J., eds. Cultura do algodoeiro. Piracicaba, Potafós, 1999. p.69-81.

TENNANT, D. A test of a modified line intersect method of estimating root length. J. Ecol., 63:995-1001, 1975.
TISDALE, S.L. \& NELSON, W.L. Fundamentals of fertilizer application. In: TISDALE, S.L. \& NELSON, W.L., eds. Soil fertility and fertilizers. 2.ed. New York, The Macmillan Company, 1966. p.499-553.

USDA-ARS. Lubuck, Texas. Cotton root disorders. Disponível em: <http://cipm.ncsu.edu/cottonpickin/disorders/>, acesso em 28 de abril de 2004. 